\title{
Wireless Body Area Network Auxiliary Relief Mechanism Based on Uneven Population Clustering and Rescue Request Information Diffusion Strategy
}

\author{
Fenglian Jiang, ${ }^{1}$ Mingling Wang, ${ }^{2}$ Chun-Chi Chen, ${ }^{3 *}$ and Hsien-Wei Tseng ${ }^{1 * *}$ \\ ${ }^{1}$ School of Mathematics and Information Engineering, Longyan University, Longyan, Fujian 364012, China \\ ${ }^{2}$ College of Artificial Intelligence, Yango University, Mawei District, Fujian 350015, China \\ ${ }^{3}$ School of Life Sciences, Longyan University, Longyan, Fujian 364012, China
}

(Received July 20, 2020; accepted March 17, 2021)

Keywords: wireless body area network, relief mechanism, uneven clustering, propagation strategy, energy consumption balance, network dynamics

We propose an auxiliary relief mechanism (ARM) for a wireless body area network (WBAN). The ARM, as a supplement to the traditional medical assistance provided by a WBAN, aims at solving the problem of the lack of timely relief in the existing WBAN relief mechanism. When an emergency occurs, it can help RSs find $k$ potential rescuers (PRs) nearby. By fully considering the limited energy consumption and dynamic characteristic of auxiliary relief network (ARN) nodes, we propose an ARN dynamic population clustering algorithm for the ARM to cluster people around RSs, which uses dynamic uneven clustering to ensure balanced energy consumption between clusters. On the basis of the clustering, a rescue request information (RRI) diffusion strategy is proposed, and appropriate intracluster and intercluster propagation strategies are adopted to spread the RRI, so that the information can be transmitted quickly to $k$ PRs. Our experiment shows that the ARN dynamic population clustering algorithm realizes uneven clustering for a dynamic population. The ARM RRI diffusion strategy is superior to a comparative algorithm in both searching time and energy consumption, and has higher searching efficiency.

\section{Introduction}

Acting as the wireless sensor network (WSN) in human monitoring applications, a wireless body area network (WBAN) has broad development prospects in medical treatment, health care, sports training, the military, aerospace, consumer entertainment, and so on, ${ }^{(1)}$ where medical treatment is the most important application scenario. A typical WBAN is composed of a set of slightly portable, communication-enabled sensors and a coordinator. Minor sensors with distinct functions can be implanted in the human body or placed on the body surface. Each sensor collects one or more body physiological information and activity signals, such as an electrocardiogram (ECG), electroencephalogram (EEG), electromyogram (EMG), temperature, motion acceleration, and angular velocity, and transmits the information to the coordinator

\footnotetext{
*Corresponding author: e-mail: 15160686635@163.com

** Corresponding author: e-mail: hsienwei.tseng@gmail.com https://doi.org/10.18494/SAM.2021.3041
} 
wirelessly. Serving as a gateway between the WBAN and the external network, the coordinator can conduct secure data collection, delivery, and exchange, so that relevant personnel can assess and diagnose the human body. ${ }^{(2-5)}$

A complete WBAN application framework typically consists of three layers. As shown in Fig. 1, the first layer is the sensing layer, which is composed of sensor nodes that monitor human physiological information. After its collection, the information is transmitted to the coordinator wirelessly. The second layer is the network layer, where the personal server (mobile phone, computer, PDA, and other smart devices) receives the data from the coordinator and saves the data to the cloud through a wireless or wired network after it is stored. The third layer is the application layer, providing a variety of applications. Using the cloud data, relevant personnel can view various physiological information of the monitored person and provide the appropriate services.

There is still a lot of room for the use of WBANs to grow in the medical field (telemedicine, special population monitoring, community medical care, etc.). The scenario of WBAN medical rescue can be described as follows. When a physical emergency (sudden physical illness, abnormal physiological signals, etc.) occurs suddenly, we can use the real-time physiological data collected by the sensor layer to judge the abnormalities of the human body. The personal server transmits the abnormal information to a medical institution through the network layer, and then the medical institution makes judgments and takes appropriate measures to implement the corresponding rescue for WBAN carriers. For WBAN carriers, the rapid response mechanism of the WBAN is extremely important when an emergency occurs. The shorter the rescue time, the better it will be for the human body. However, in reality, the traditional WBAN rescue method cannot provide timely assistance to patients. For example, the increase in the aged population has increased the pressure on medical resources (medical institutions, medical staff, medical devices, etc.), and the relevant medical staff cannot be arranged in a timely manner for rescue seekers (RSs). If the medical institution is far away and the rescue process lasts for a long time, then the emergency of an RS cannot be handled immediately, which may cause irretrievable

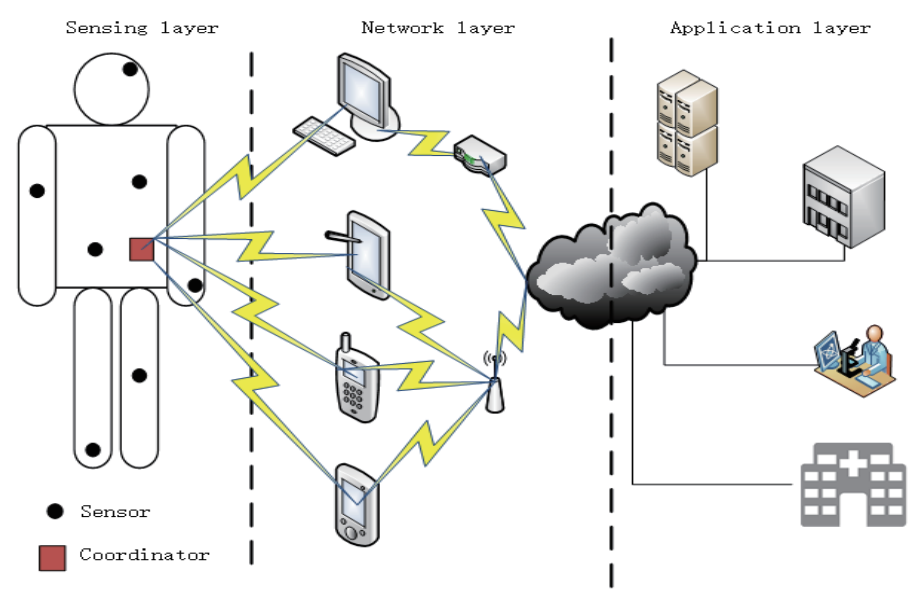

Fig. 1. (Color online) WBAN application framework. 
consequences; if the RS does not have a smart device at hand, which can send a signal to the network, information on an abnormality may be unable to reach the medical institution.

So that WBANs can play a greater role in the medical field, we have designed an auxiliary relief mechanism (ARM, Fig. 2) for a WBAN for telemedicine assistance under certain assumptions. When identifying the emergency of a human body through data collected by the sensor, the WBAN will send rescue request information (RRI) to the telemedicine assistance center, start the ARM rapid response, and make use of the WBAN coordinator to build an auxiliary relief network (ARN). By employing various strategies, we can find $k$ potential rescuers (PRs) in the ARN and provide timely medical assistance to RSs.

When designing the ARM in this study, we use the WBAN's coordinator to construct the ARN and to propagate RRI. In essence, the coordinator is still a sensor; thus, the systems and characteristics of the sensor need to be considered, such as the limited propagation distance and the control of energy consumption. The nodes in the ARN have limited energy and a limited communication distance. At the same time, the physical location of the WBAN will change with the movement of the human body, so the nodes in the ARN are dynamic.

In past research, the traditional flooding algorithm (or broadcast algorithm) has not been applied to finding $k$ PRs quickly in a crowd; because the diffusion of RRI leads to excessive data communication, the speed of finding key nodes is too low and too much energy is consumed. Under the condition that each coordinator has balanced energy consumption, we can quickly transmit the RRI issued by the RS to $k$ PR nodes. However, in this study, we refer to the knowledge of the clustering routing protocol to achieve the ARM. We consider and compare the approach proposed to achieve the ARM with the approach proposed by Heinzelman et al. ${ }^{(5)}$ The clustering routing protocols have uniform and non-uniform approaches. The LEACH protocol is a typical uniform clustering routing protocol. This protocol adopts a random clustering strategy and cluster-head cycle rotation strategy. As a result, the clusters are equal in size with the same number of members. Although the protocol adopts the cluster-head cycle rotation strategy, the

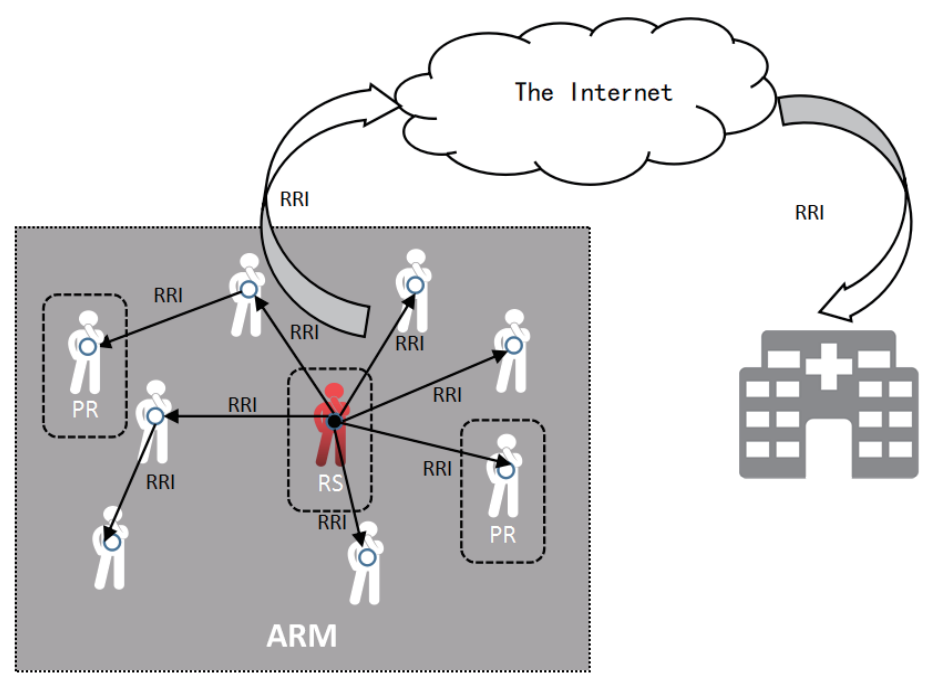

Fig. 2. (Color online) Schematic diagram of ARM. 
cluster head nodes far from the base station will consume more energy because of their singlehop transmission of information, which cannot guarantee the balanced energy consumption of each node. If a multihop method is used to propagate the message in a uniform clustering network, then the cluster head nodes near the base station will undertake more data-forwarding tasks, which also cannot guarantee balanced energy consumption. As a result, uneven clustering occurs. For example, the EECS ${ }^{(6)}$ uneven clustering protocol uses single-hop communication, where the clusters further from the base station have a smaller radius. The $\operatorname{UCS}^{(7)}$ protocol uses multihop communication to dynamically adjust the size of the clusters according to the cluster head to balance the energy consumption of the nodes. The DEBUC ${ }^{(8)}$ protocol combines uneven clustering and intercluster multihop routing, which achieves an efficient energy balance. The choice of the cluster head is based on the residual energy of candidate cluster heads and neighbor nodes. Among the clusters generated by this protocol, the radius of a cluster closer to the base station is smaller. Other uneven clustering protocols include EEUC ${ }^{(9)}$ and ACOUC. ${ }^{(10)}$

Although the above uneven clustering protocols can guarantee the balanced energy consumption of the nodes, the sensor networks in these studies were stable, that is, the positions of the nodes were fixed. For the clustering of the ARN, it is necessary to consider that the nodes will move randomly with the movement of the human body, namely, the dynamics of the node must be considered in addition to the characteristics of the traditional sensor network, such as limited energy consumption and limited communication distance. Therefore, as the novelty of this study, the ARN dynamic crowd clustering algorithm takes into account the dynamics of nodes in the selection of cluster heads and uses a nonuniform clustering method to ensure balanced energy consumption between clusters. Then, an RRI diffusion strategy based on the clustering is proposed, in which appropriate intracluster and intercluster propagation strategies are used to help spread the RRI sent by the RS. The information can be quickly propagated to $k$ PRs, the RRI of the RS is notified to the PRs, and the ARM is realized.

\section{Materials and Methods}

\subsection{ARN model and model assumptions}

It is assumed that at a certain moment, $n$ WBANs are randomly distributed in a certain space. When an emergency occurs, the ARM starts. For the nodes of each WBAN coordinator corresponding to the ARN, $v_{i}$ represents the $i$ th node and the corresponding node set is $V=\left\{v_{1}, v_{2}, \ldots, v_{n}\right\}$. We assume that the ARN application scenario studied has the following characteristics:

(1) Each node has a unique physical identity and can be identified uniquely.

(2) Two nodes can communicate with each other within the communication interval.

(3) The nodes have the function of data storage and possess the basic personal information of carriers.

(4) The nodes are dynamic, that is, the node positions are not fixed.

(5) Each mode is isomorphic and can act as a cluster head or a member node, and the initial energy of each node is limited. 
(6) The power of the nodes can be adjusted. The power of the transmitted signal can be adjusted according to the distance from the nodes, and they can also calculate the distance between nodes from the strength of the received signal.

(7) Nodes can reduce the amount of data transmission through data fusion technology.

\subsection{ARN energy consumption model}

As the energy of the coordinator is limited, the energy consumption of the ARN should be considered, most of which is for communication, with much smaller amounts used for perception and calculation. Therefore, we use a simple energy consumption model ${ }^{(11)}$ to characterize the energy consumption in the ARN, ignoring the energy consumption of the nodes in operation, storage, or sleep. Node energy is consumed in the transmitter and receiver. Under the premise of improving the signal-to-noise ratio, the energy consumption assumed for data transmission is

$$
E_{T x}(l, d)= \begin{cases}l E_{\text {elec }}+l E_{f s} d^{2}, & d<d_{0} \\ l E_{\text {elec }}+l E_{m p} d^{4}, & d \geq d_{0}\end{cases}
$$

and that for receiving data is

$$
E_{R x}(l)=l E_{\text {elec }},
$$

where $E_{T x}$ is the energy consumption for data packet transmission, $E_{R x}$ is the energy consumption for receiving a data packet, each member node sends $l$ bits of data, $E_{\text {elec }}$ is the energy consumption for transmitting and receiving one bit of data, $E_{f s}$ and $E_{m v}$ are the energy consumption coefficients of the circuit power amplifier in different channel transmission models, $d_{0}$ is the transmission distance threshold of the sensor nodes, and $d$ is the transmission distance.

\subsection{ARN data fusion model}

Using a data fusion model in the process of data transmission can effectively reduce the amount of data transmission, thus reducing the total energy used by the network and increasing the lifetime of the network. ${ }^{(12)}$ As the data transmission between clusters is clearly different for each transmission, we only consider data fusion in a cluster, instead of that between clusters. The data fusion model in the cluster assumes that when cluster head nodes receive one bit of data sent by each node in the cluster, the data is compressed into one bit of data, regardless of the number of nodes in the cluster, and the energy consumption is set as $E_{D}=5 \mathrm{~nJ} / \mathrm{bit}$.

\section{ARN Dynamic Population Clustering Algorithm}

If the number of nodes in the cluster and the geometric size of the cluster are too large or too small and the shape of the set is too large or too small, the energy consumption will not be 
optimal; therefore, the ARN dynamic population clustering protocol adopts uneven clustering to ensure balanced energy consumption of the network nodes. Also, each cluster has a reasonable radius to ensure the balanced energy consumption of the internal network nodes, so as to maximize the network lifetime.

\subsection{Selection of the candidate cluster head}

In the ARN dynamic population clustering algorithm, the selection of cluster head nodes is based on local competition between the nodes, and the nodes compete to be the cluster head according to certain rules. Because of the limited energy consumption and dynamic characteristics of ARN nodes, we consider the residual energy and stability of nodes in the selection of candidate cluster heads. First, we define the stability of a node in the ARN.

Definition 1: In the ARN, the stability of a node is the distance that the node moves within a fixed time interval.

Assuming that the distance node $v_{i}$ moves within a fixed time interval $\Delta T$ is $d_{\Delta T}$, the stability of $v_{i}$ is defined as

$$
S_{v_{i}}=\frac{\Delta T}{d_{\Delta T}}
$$

That is, the further the node moves within $\Delta T$, the more unstable the node and the smaller the stability $S_{v_{i}}$.

In the selection of the cluster head, it is not necessary for every node to become a candidate cluster head. Thus, in the selection of candidate cluster heads, each node generates a random number $u$ between 0 and 1 , and if $u$ is less than a threshold $T(i)$, then it becomes a candidate cluster head node, where

$$
T(i)=\frac{E_{v_{i}}}{E_{0}} S_{v_{i}} \omega
$$

Here, $E_{v_{i}}$ is the residual energy of the node, $E_{0}$ is the initial energy of the node, and $\omega$ is the ratio of the number of candidate cluster heads to the total number of nodes, which is used to control the number of candidate cluster heads to a reasonable range. From Eq. (4), we can see that a node with more residual energy and greater stability is more likely to be a cluster head node.

\subsection{Radius of the candidate cluster head}

Each candidate cluster head has a certain radius $R_{v_{i}}^{\text {compete }}$ to control the size of the cluster, and the radius of the cluster involves the balance of the distance and the energy; therefore, $R_{v_{i}}^{\text {compete }}$ is a function comprehensively considering the distance and the energy. To balance the energy consumption, a cluster closer to the RS node or with less residual energy has a smaller radius, 
and $R_{v_{i}}^{\text {compete }}$ is proportional to $d\left(v_{i}, R S\right)$ and $E_{v_{i}}$. Thus, the radius of the candidate cluster head node $v_{i}$ is obtained using the following formula.

$$
R_{v_{i}}^{\text {compete }}=\left(1-\alpha \frac{d_{\max }-d\left(v_{i}, R S\right)}{d_{\max }-d_{\min }}-\beta \frac{E_{0}-E_{v_{i}}}{E_{0}-E_{\text {emp }}}\right) R^{0}
$$

Here, $d_{\max }$ and $d_{\min }$ are the maximum distance and minimum distance of the node to the RS node, respectively; $d\left(v_{i}, R S\right)$ is the distance from node $v_{i}$ to the $R S$ node; $E_{0}, E_{v_{i}}$, and $E_{\text {emp }}$ are respectively the initial energy of the node, the current residual energy of the node, and the transmission energy threshold of the node; $R^{0}$ is the maximum transmission radius of the node; and $\alpha$ and $\beta$ are both constants in the interval $[0,1]$.

\subsection{Clustering process}

After calculating the cluster radius and the candidate cluster head nodes, the cluster head nodes are selected by the following process:

(1) Select the candidate node

Generate a random number $u$ between 0 and 1 . If $u$ is less than $T(i)$, then the candidate node becomes a candidate cluster head node and broadcasts the node message CompSendMsg (ID, $R_{v_{i}}^{\text {compete }}, E_{v_{i}}$ ). If $u$ is greater than $T(i)$, then it becomes a normal node, quits the process of cluster head selection, and enters the sleep mode until the cluster head selection is finished.

(2) Determine the information list of the neighbor candidate cluster head nodes

After the candidate cluster head nodes are determined, each node determines the information list of its neighbor cluster head nodes.

(3) Determine the cluster head node

If the residual energy $E_{v_{i}}$ of a candidate cluster head node is larger than the average energy $\bar{E}_{\text {NbNode }_{j}}$ of a neighbor cluster head node, the $f_{v_{i}}$ value of each cluster head node in the neighbor list is calculated. The candidate cluster head node with the highest $f_{v_{i}}$ value is selected as the cluster head node. $f_{v_{i}}$ is calculated as

$$
f_{v_{i}}=\frac{E_{v_{i}} S_{v_{i}}}{d\left(v_{i}, R S\right)} .
$$

It can be seen that the cluster head nodes are the nodes with a large energy, high stability, and small distance.

(4) The cluster head node broadcasts a message and other nodes join the cluster.

After the cluster head node $v_{i}$ is determined, it sends the message FinalHeadMsg(ID) to the common nodes within the clustering radius of $R_{v_{i}}^{\text {compete }}$. After each node receives the message, it returns the clustering message AddClusterMsg(ID) to the cluster head node. With this, the uneven clustering is completed. Algorithm 1 is the pseudo-code of the clustering process. A schematic diagram of the clustering result is shown in Fig. 3. 

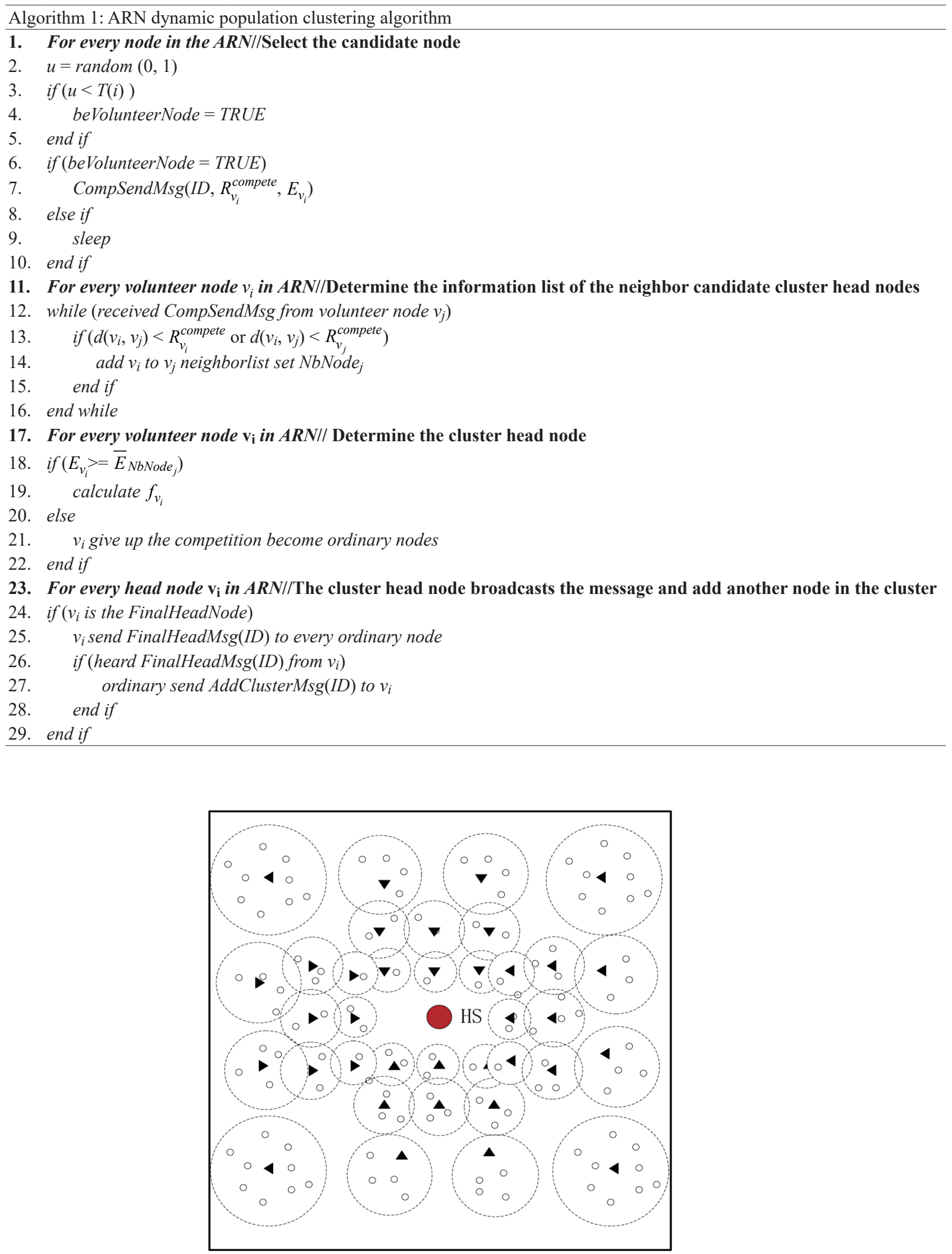

Fig. 3. (Color online) Schematic diagram of the clustering result. 


\section{RRI Diffusion Strategy}

\subsection{Node information table}

Each node has a personal information table that stores the ID of the node, the residual energy, the distance to the RS, whether it is a PR, and so on. Each cluster head node not only has a personal information table, but also maintains a member information table and a neighbor cluster head information table. The member information table stores two items: the ID of nodes in the cluster and whether each node is a PR. The neighbor cluster head information table contains the information of the neighbor cluster head node, such as the node ID, the residual energy, the distance to the RS, and so on.

\subsection{Diffusion strategy}

The ARM adopts a single-hop information diffusion method in the cluster and a multihop method between the clusters. The single-hop diffusion in the cluster means that the cluster head broadcasts messages to the nodes in the cluster. The information diffusion between clusters is conducted by multihop diffusion, so an intercluster diffusion strategy is needed to find the optimal next-hop cluster head. During the intercluster diffusion, the current cluster head node selects an optimal cluster head as the next-hop forwarding node from the set of neighbor cluster head nodes according to a certain selection rule. To quickly propagate the messages to the key nodes while maintaining the stable propagation of messages and balanced energy consumption, we need to consider three factors: the distance of the next-hop cluster head, the stability of the nodes, and the energy.

(1) The energy weight formula of cluster head node $v_{i}$ transmitting messages to the neighbor cluster head node $v_{j}$ is

$$
P E\left(v_{j}\right)=\frac{E_{v_{j}}}{E_{\max }},
$$

where $E_{v_{j}}$ is the residual energy of the neighbor cluster head node $v_{j}$ and $E_{\text {max }}$ is the maximum value of $E_{v_{j}}$ among the neighbor cluster head nodes $v_{j}$.

(2) The distance weight is an important parameter for evaluating the propagation speed of the nodes. The distance weight formula of nodes is

$$
P D\left(v_{j}\right)=\frac{d\left(v_{j}, R S\right)}{\sqrt{d^{2}\left(v_{i}, v_{j}\right)+d^{2}\left(v_{j}, R S\right)}},
$$

where $d\left(v_{j}, R S\right)$ is the distance from the next-hop cluster head node to the RS and $d\left(v_{i}, v_{j}\right)$ is the distance from the current node to the next-hop node. 
(3) The stability of node $v_{j}$ is given by

$$
P S\left(v_{j}\right)=\frac{S\left(v_{j}\right)}{S_{\max }},
$$

where $S\left(v_{j}\right)$ is the stability value of the next-hop node and $S_{\max }$ is the maximum stability value among the next-hop candidate cluster head nodes.

To sum up, we define the selection function of the next-hop cluster head $v_{j}$ as

$$
P\left(v_{j}\right)=\lambda_{1} P E\left(v_{j}\right)+\lambda_{2} P D\left(v_{j}\right)+\lambda_{3} P S\left(v_{j}\right)
$$

where $\lambda_{1}, \lambda_{2}$, and $\lambda_{3}$ are weight coefficients and $\lambda_{1}+\lambda_{2}+\lambda_{3}=1$.

The above selection function indicates that a node with higher residual energy that is closer to the RS and of higher stability will be preferred as the next-hop cluster head node, which ensures that the information transmission is fast and effective.

\section{ARM}

(1) When the WBAN detects abnormal physiological parameters of the carrier, the ARM starts. The RS, being the RS node, builds the ARN network and implements the dynamic population clustering algorithm to cluster the people around the RS. After the clustering is completed, the RS finds the cluster head node of the largest $P\left(v_{j}\right)$ value through Eq. (10) and sends RRI to the cluster head node at the same time.

(2) The cluster head node that receives the RRI checks whether it is a PR node. If so, it jumps to the count attribute, and one is added to the count value. The count value is compared with $k$, and if it is less than $k$, the RRI message is first diffused to the cluster member nodes.

(3) When the cluster member nodes receive the RRI, they check whether they are PR nodes or not. If they are not PR nodes, they send a NO message to the cluster head node. If they are PR nodes, they send a YES message to the cluster head node. At the same time, one is added to the count attribute value of the cluster head node, and the cluster head node jumps to the count attribute.

(4) If the count attribute value of the cluster head node is less than $k$, then the RRI and count are sent to the neighbor cluster head node with the highest priority in the neighbor cluster head table, and the neighbor cluster head node repeats the above steps (2) and (3).

(5) If the count attribute value of the cluster head node is equal to $k$, then the diffusion is over.

\section{Results and Discussion}

\subsection{Experimental settings}

We simulated the implementation of the ARM on the Python environment and compared multiple performances of the ARM. In the experiment, 1000 individuals were randomly 
distributed in a square area of $400 \times 400 \mathrm{~m}^{2}$ and the number of PSs was 50 . The RS node was located in the center of the square, namely, at the coordinates $(200,200)$, and the communication between the sensors adopted the ARN energy consumption model described in Sect. 2.2. The relevant parameters in the experiment are shown in Table 1.

\subsection{Analysis of results}

\section{(1) Clustering}

Figure 4 shows the context diagram of the node's competing radius $R_{v_{i}}^{\text {compete }}$, the distance from the node to the RS, $d\left(v_{i}, R S\right)$, and the residual energy of the node, $E_{v_{i}}$. It can be seen from the figure that the competing radius $R_{v_{i}}^{\text {compete }}$ of the node increases as the distance from the node to the RS, $d\left(v_{i}, R S\right)$, increases, and the competing radius becomes smaller with decreasing $E_{v_{i}}$. This guarantees the energy balance of all the cluster heads.

Figure 5 shows the cluster head distribution map obtained by the dynamic population clustering algorithm in the experiment. It can be seen that the closer the distance to the RS node, the greater the density of cluster heads.

Table 1

Parameter settings.

\begin{tabular}{lcccc}
\hline Parameter & Value & & Parameter & Value \\
\hline RS Coordinates & $(100,100) \mathrm{m}$ & & Area Size & $(0,0)-(400,400) \mathrm{m}$ \\
$E_{f s}$ & $9.5 \mathrm{pJ} /\left(\mathrm{bit} \times \mathrm{m}^{2}\right)$ & & $E_{0}$ & $0.5 \mathrm{~J}$ \\
$E_{m p}$ & $0.0053 \mathrm{pJ} /\left(\mathrm{bit} \times \mathrm{m}^{2}\right)$ & $\Delta T$ & $10 \mathrm{~s}$ \\
$\alpha$ & 0.6 & $\omega$ & 0.15 \\
$\beta$ & 0.4 & $\lambda_{1}$ & 0.3 \\
$E_{\text {emp }}$ & $0.1 \mathrm{~J}$ & $\lambda_{2}$ & 0.4 \\
$R^{0}$ & $20 \mathrm{~m}$ & $\lambda_{3}$ & 0.3 \\
$E_{\text {elec }}$ & $50 \mathrm{~nJ} / \mathrm{bit}$ & $E_{D}$ & $5 \mathrm{~nJ} / \mathrm{bit}$ \\
\hline
\end{tabular}

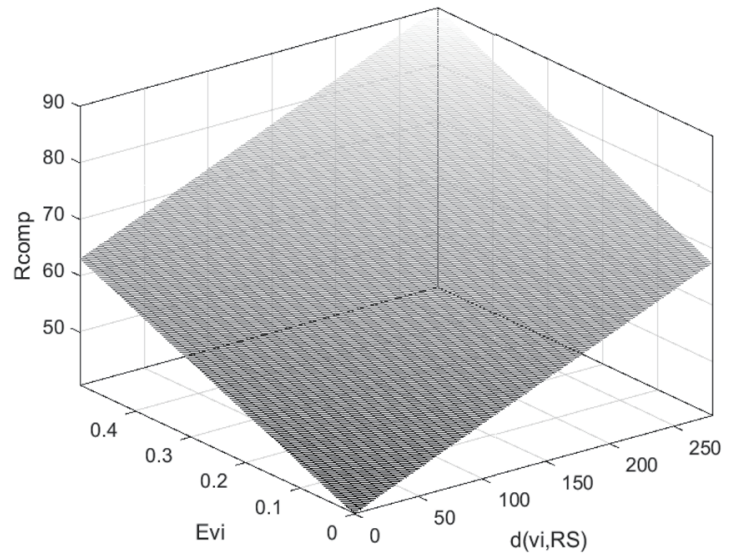

Fig. 4. Diagram showing relationship between radius, distance, and energy of node.

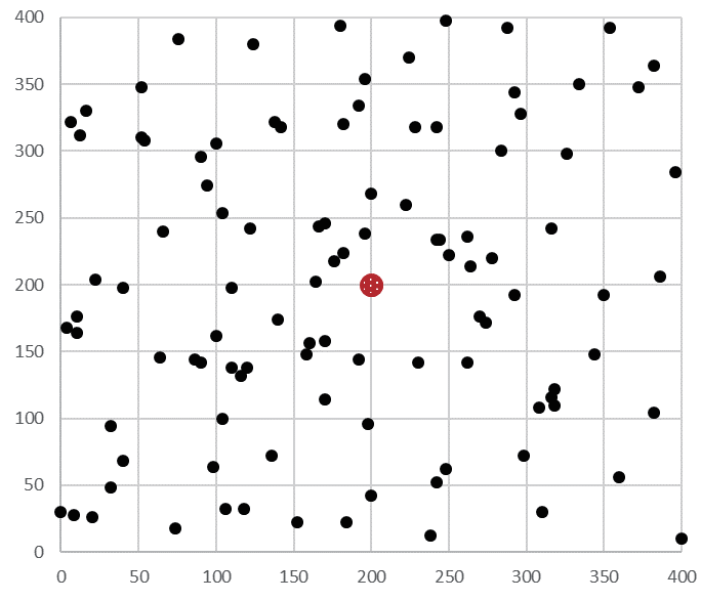

Fig. 5. (Color online) Distribution of cluster heads. 
(2) Searching efficiency

The design goal of the ARM is to find $k$ PR nodes quickly under the condition of balanced energy consumption. In the experiment, the searching efficiency was mainly measured in terms of searching time and energy consumption. During the experiment, we compared the RRI diffusion strategy of the ARM with that of the nearest-distance matching algorithm (NDMA). There is no clustering in the NDMA, and the node selects the node closest to it as the next-hop node.

In the experiment, it was assumed that it takes one unit of time for the RRI to transmit from one node to another each time (where it takes one unit of time to transmit between the cluster head nodes, as well as from the cluster head to the cluster nodes). Figure 6 shows a comparison of the searching time for finding $k$ PRs between the ARM RRI diffusion strategy and the NDMA. It is assumed that the transmission of RRI from one node to another costs one unit of energy each time (where the transmission between the cluster head nodes costs one unit of energy, and the energy consumption of the transmission from the cluster head to the cluster nodes is calculated from the number of nodes). Figure 7 shows the energy consumption of the ARM RRI diffusion strategy and the NDMA in searching for $k$ PRs.

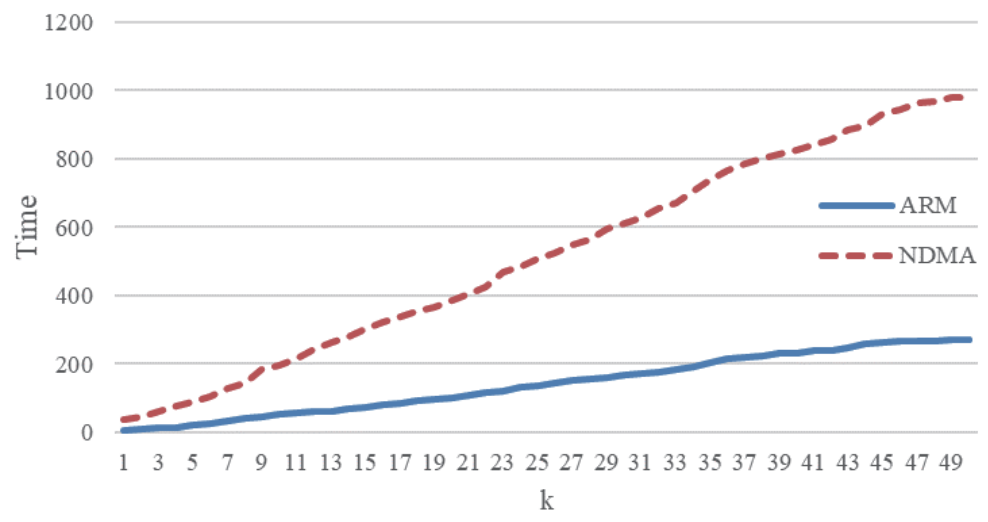

Fig. 6. (Color online) Time to search for different numbers of PRs.

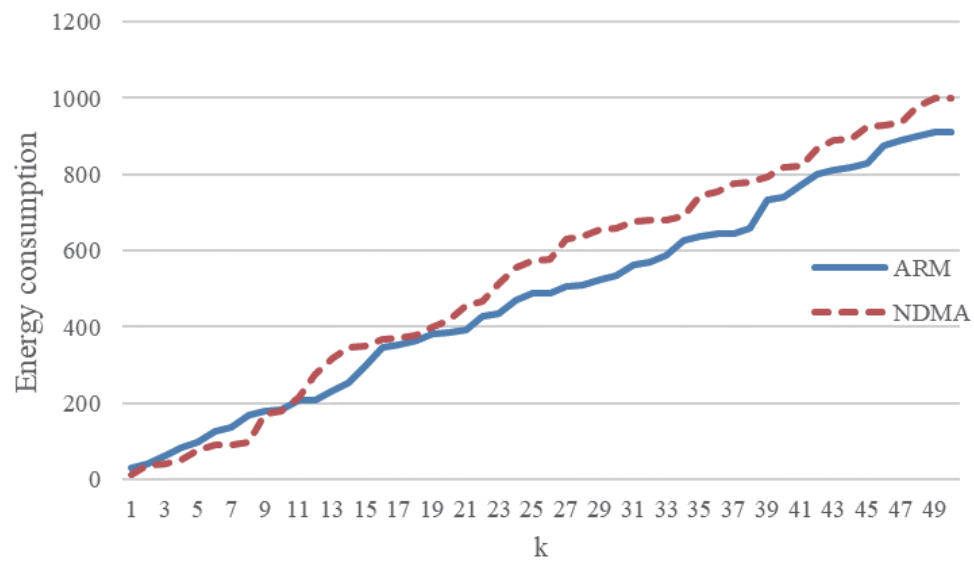

Fig. 7. (Color online) Energy consumption for searching for different numbers of PRs. 
From the experimental results, we can see that with increasing number of PRs, that is, with increasing $k$, the searching time of the two algorithms increases gradually along with the energy consumption. In terms of the searching time, as the RRI diffusion strategy adopts single-hop diffusion in the cluster and multihop diffusion between the clusters on the basis of the clustering, it finds the PR nodes faster than the NDMA algorithm, as shown in Fig. 6. From Fig. 7, we can see that the ARM RRI diffusion strategy consumes slightly more energy than the NDMA when finding a smaller number of PR nodes, but with increasing number of PR nodes, the energy consumption gradually becomes less than that of the NDMA. This indicates that the RRI diffusion strategy can effectively reduce the energy consumption during RRI propagation, as well as reduce the energy consumption of the ARM mechanism.

Therefore, the ARM RRI diffusion strategy in this paper has better performance than the NDMA in searching time and energy consumption, which shows that it has greater searching efficiency.

\section{Conclusion}

Because the existing WBAN medical assistance mechanism may not be able to perform an emergency rescue in time, we proposed a WBAN ARM to help a RS find $k$ PRs in a nearby crowd when an emergency occurs. The ARM makes use of the coordinator of a human WBAN to build an ARN. We proposed a dynamic population clustering algorithm for the ARN based on the characteristics of limited energy consumption and dynamic nodes. It clusters the people around the RS and uses dynamic uneven clustering to ensure the balanced energy consumption of each node. On the basis of the clustering, we propose an RRI diffusion strategy: the RRI is diffused through single-hop information diffusion in the cluster and multihop information diffusion between the clusters, so that the information can be transmitted efficiently to $k$ PRs. The experiment shows that the ARN dynamic population clustering algorithm realizes uneven clustering for a dynamic population and provides a foundation for RRI diffusion. As a result of such clustering, the ARM RRI diffusion strategy is superior to the NDMA in both searching speed and energy consumption, indicating its higher PR searching efficiency.

\section{Acknowledgments}

This work was supported by Science and Technology Project of Longyan City under Grant (2017LY74) and Longyan University's Qi Mai Science and Technology Innovation Fund Project of Longyan City (2017QM0201 and 2018LYQM0202), Longyan University's Qi Mai Science and Technology Innovation Fund Project of Liancheng ([2018]132) and Shanghang (2019SHQM05) County, Longyan and Longyan University's Research and Development Team Fund ((2018)8), and Great Project of Production, Teaching, Research of Fujian Provincial Science and Technology Department (2019H6023). 


\section{References}

1 J. Gong, R. Wang, and L. Cui: J. Comput. Res. Dev. 47 (2010) 737. http://crad.ict.ac.cn/EN/Y2010/V47/I5/737

2 B. Latré, B. Braem, I. Moerman, C. Blondia, and P. Demeester: Wireless Networks 17 (2011) 1. https://doi. org/10.1007/s11276-010-0252-4

3 R. Cavallari, F. Martelli, R. Rosini, C. Buratti, and R. Verdone: IEEE Commun. Surv. Tutorials 16 (2014) 1635. https://doi.org/10.1109/SURV.2014.012214.00007

4 Y. Liu and Y. Song: J. Chin. Comput. Syst. 34 (2013) 1757. https://doi.org/10.3969/j.issn.1000-1220.2013.08.010

5 W. R. Heinzelman, A. Chandrakasan, and H. Balakrishnan: Proc. 33rd Annu. Hawaii Int. Conf. System Sciences (IEEE, 2000). https://doi.org/10.1109/HICSS.2000.926982

6 G. Chen, C. Li, M. Ye, and J. Wu: J. Front. Comput. Sci. Technol. 1 (2007) 170. https://doi.org/10.3778/j. issn.1673-9418.2007.02.005

7 S. Soro and W. B. Heinzelman: 19th IEEE Int. Parallel and Distributed Processing Symp. (IEEE, 2005). https:// doi.org/10.1109/IPDPS.2005.365

8 C. Jiang, W. Shi, X. Tang, P. Wang, and M. Xiang: J. Software 23 (2012) 1222. https://doi.org/10.3724/ SP.J.1001.2012.04061

9 C. Li, M. Ye, G. Chen, and J. Wu: IEEE Int. Conf. Mobile Adhoc and Sensor Systems Conf. (IEEE, 2005) 604. https://doi.org/10.1109/MAHSS.2005.1542849

10 J. Du and L. Wang: 2011 3rd Int. Conf. Comput. Res. Dev. (IEEE, 2011) 67-71. https://doi.org/10.1109/ ICCRD.2011.5764247

11 C. Intanagonwiwat, R. Govindan, D. Estrin, J. Heidemann, and F. Silva: IEEE/ACM Trans. Networking 11 (2003) 2. https://doi.org/10.1109/TNET.2002.808417

12 X. Liu, F. Li, H. Kuang, and X. Wu: 2006 6th World Congr. Intell. Control Autom. (IEEE, 2006) 5120-5124. https://doi.org/10.1109/WCICA.2006.1713366 